\title{
Anal Extrusion of a Ventriculoperitoneal Shunt
}

\author{
Omer Furkan Turkis1, Ali Karadag1, Erik H. Middlebrooks² and Mehmet Senoglu1
}

\begin{abstract}
Ventriculoperitoneal shunt (VPS) placement is an effective and most frequently used surgical method in the treatment of hydrocephalus, but the mechanical and infective complications are often seen after this surgical procedure. Bowel perforation after VPS surgery is rarely seen complication that is reported ranging between $0.1 \%$ and $0.7 \%$ in the literature. We report a case of 10-month baby who was shunted at day three of her life and has presented to us with protruding distal end of the ventricular catheter through anus. Mechanism of migration of VPS is unclarified yet; nevertheless, children with myelomeningocele have weakness of the bowel muscles, which probably makes it more sensitive for perforation. Additionally, sharp and stiff end of the VPS, use of trocar by some surgeons, chronic irritation by the shunt, previous surgery, infection and silicone allergy are other possible reasons of bowel perforation. Peritonitis and ventriculitis have a high morbidity and mortality that may occur after VPS-related bowel perforations; hence, it should be managed rapidly and aggressively to reduce morbidity and mortality.
\end{abstract}

Key Words: Ventriculo-peritoneal shunt, Perforation, Hydrocephalus.

\section{INTRODUCTION}

Ventriculoperitoneal shunt (VPS) placement is an effective and widely used treatment of hydrocephalus. 1 Various complications of VPS have been reported in the literature that can be generally divided into mechanical and infectious complications. Typical complications include shunt obstruction, disconnection, or migration of any components of the shunt system, either at the ventricular or peritoneal end. Infectious complications include shunt tract abscess, skin necrosis overlying the shunt device and ventriculitis. Others have also been described, such as seizures, subdural collections, craniosynostosis, inguinal hernia, hydrocele, ascites, pseudocyst formation, perforation of a viscus or extrusion of the shunt and intestinal volvulus. ${ }^{2}$

The majority of the complications are associated with the cranial part, up to $25 \%$ involve the abdominal part. The incidence of the bowel perforation has been reported as varying from $0.1-0.7 \%$ after VPS surgery and has a high mortality rate $(15 \%) .3-5$

We present a case of VPS bowel perforation and extrusion through the anus as a rare, but important complication to be recognised.

${ }^{1}$ Department of Neurosurgery, Tepecik Research and Training Hospital, University of Health Sciences, Izmir, Turkey

2 Radiology and Neurosurgery, Mayo Clinic, Jacksonville, FL, USA

Correspondence: Dr. Mehmet Senoglu, Department of Neurosurgery, Tepecik Research and Training Hospital, University of Health Sciences, Izmir, Turkey

E-mail:mehmetsenoglu@hotmail.com

Received: August 16, 2018; Accepted: October 20, 2018

\section{CASE REPORT}

A 10-month girl with a history of shunt placement at day three of her life for congenital hydrocephalus presented with intractable vomiting. Her VPS catheter reportedly had been protruding through anus (Figure 1A).

On admission, she was drowsy and hypoactive, with no signs of raised intra-cranial pressure, as there were no "sunset eyes" sign and the anterior fontanelle was normal. Furthermore, there was no fever nor meningeal irritation signs. Her abdomen was soft with no rebound tenderness. The patient underwent computerised tomography (CT) scan of the head showing no progressive ventricular enlargement compared to prior examinations (Figures

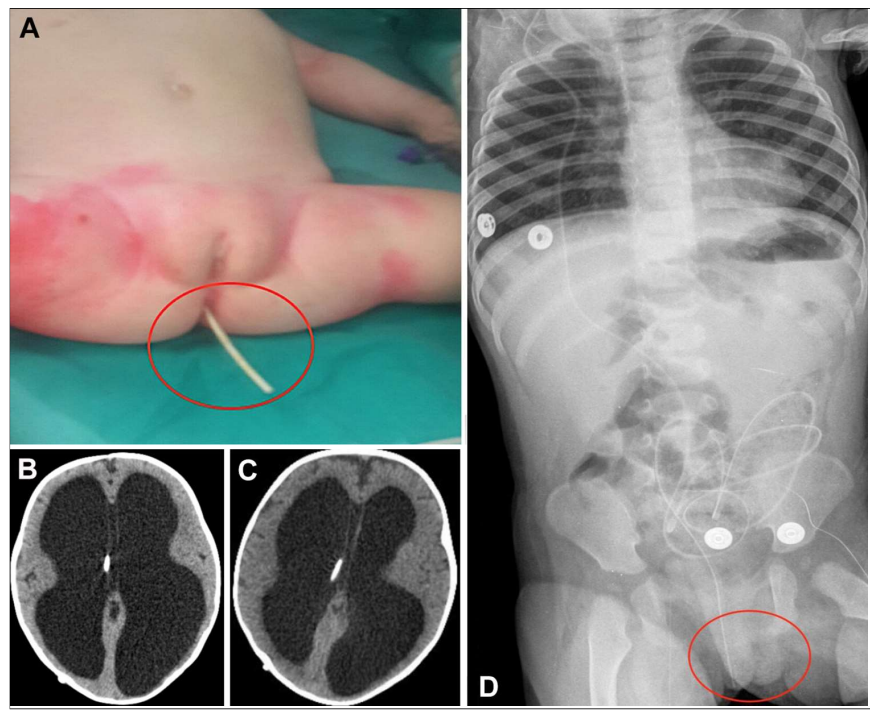

Figure 1: (A) The photograph is showing protruding of the distal catheter end through the anus. $(\mathbf{B}, \mathbf{C})$ Brain computed tomography scan is indicating ventricular enlargement. Proximal tube of VPS is demonstrating 3 months before the surgery and before 3 hours prior to revision surgery on the CT. (D) The shuntogram is exhibiting the migration of distal tube of VPS through the anus. 


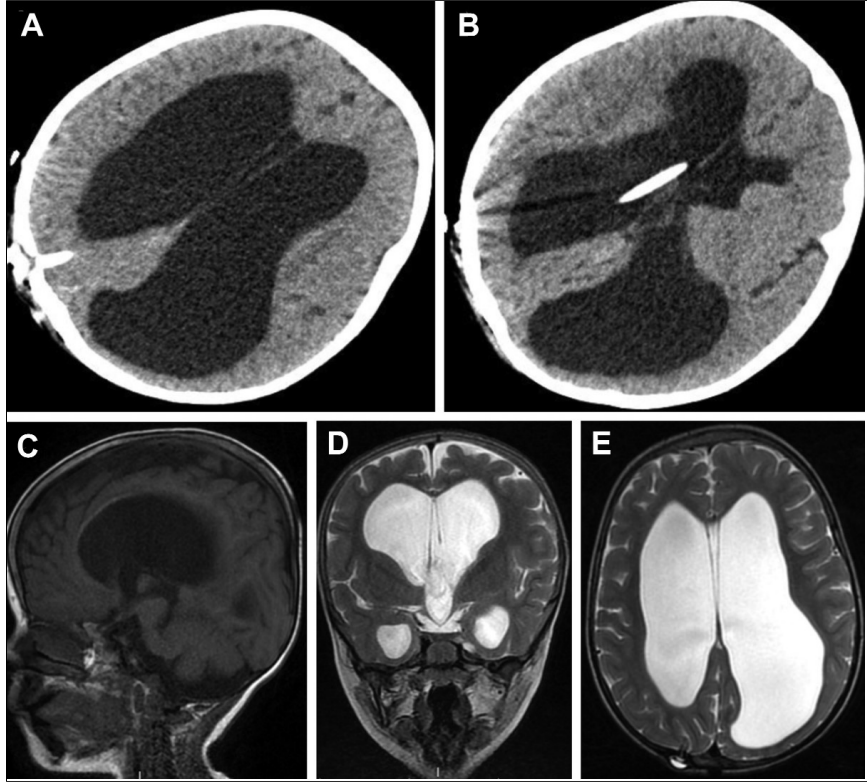

Figure 2: Brain CT (A, B) and $\mathrm{MRI}(\mathbf{C}, \mathbf{D}, \mathrm{E})$ at 2-year interval showing regression of hydrocephalus and recovery of brain tissue.

1B and 1C). There was no organised fluid collection within the abdomen on abdominal ultrasound. There was no disconnection or kinking of the shunt on a shuntography (Figure 1D).

The initial operation was performed to separate the distal end of the catheter just below the reservoir. Next, the distal catheter was removed through the rectum and an external ventricular drain was placed after removing the ventricular end. Cerebrospinal fluid (CSF) and the ventricular portion of the shunt were sent for culture, both showed growth of Escherichia coli. The patient was placed on a course of intravenous antibiotic treatment before shunt re-implantation. The patient was followed by pediatric general surgery regarding the abdominal complications. The patient was discharged without complications three days after shunt re-implantation. She is in a good condition at the 2-year internal and brain CT (Figures 2A and 2B) and MRI (Figures 2C, 2D and $2 \mathrm{E}$ ) showed the regression of the hydrocephalus.

\section{DISCUSSION}

VPS is a well-established surgical treatment for hydrocephalus. While VPS has become a somewhat routine neurosurgical procedure, complications continue to occur. Of these, as many as $25 \%$ of the complications, involve the abdominal portion of the VPS; however, bowel perforation is relatively rare with an incidence between $0.1-0.7 \%$. Although infrequent, bowel perforation must be recognised, as a delay in diagnosis may have significant morbidity and mortality related to peritonitis and ventriculitis. ${ }^{3}$

Peritoneal catheter perforations into the gastrointestinal system have been reported most commonly into the large bowel $(70 \%)$, stomach $(16 \%)$, and small bowel
(14\%). ${ }^{3}$ Bowel perforations may present acutely with peritonitis and typically require surgical intervention in the abdomen to repair the perforation. As in our patient, chronic perforations can be more insidious in onset with patients not showing typical signs of peritonitis. In these cases, VPS should be removed to prevent bacterial migration and ventriculitis. The mechanism of delayed bowel perforation associated with VPS is uncertain and likely multifactorial. Bowel perforations may occur due to the stiff end of the VPS, trocar injury, chronic irritation by the shunt, previous surgery, infection and silicone allergy. 6 Additionally, it has been suggested that neurologic deficits may also predispose to perforation, such as in myelomeningocele cases, due to weakness of the intestinal muscles allowing easier penetration by the VPS catheter.7,8

Acute symptoms, such as abdominal pain, vomiting, and fever are present in $44 \%$ of cases of shunt-related bowel perforations; however, over half of patients are asymptomatic.7,8 Clinical signs of meningitis are present in half of patients with chronic VPS bowel perforation. In most cases, radiographs and CT are necessary for accurate diagnosis. $5,7,9$ If there is a high suspicion about bowel perforation, injection of iodinated contrast material through the valve may assist in identification of the site of perforation on CT or radiograph. 10

In this patient, both ventricular end of VPS and CSF cultures showed growth of Escherichia coli. The plan was intravenous antibiotic treatment until CSF culture and CSF biochemistry results were for suitable re-shunting. Vancomycin and ceftriaxone were utilised empirically for two weeks. When CSF culture grew Escherichia coli, ceftriaxone was exchanged with ceftazidime and the antibiotics treatment was continued for a total of three weeks. After three weeks in hospital, CSF culture was sterile and CSF parameters were suitable for re-shunting. During the interval between removal of shunt and its replacement, external ventricular drainage was utilised for limiting hydrocephalus. The patient was followed up by pediatric surgeons about abdominal complications daily. Following suitable CSF results for shunt surgery, the child was taken for VPS operation. She was discharged three days after the operation.

Treatment of VPS-related bowel perforations is dependent on the site of perforation and patient's signs and symptoms. Extra-peritoneal perforations, such as of distal colon, may be locally contained and not produce typical peritoneal symptoms. As such, these perforations may present in the chronic phase and surgical repair of the abdominal portion may not be required. Intraperitoneal bowel perforations may present more acutely with signs and symptoms of peritonitis. If there is concern for peritonitis or possible abscess formation, these patients may require urgent surgical intervention 
in the abdomen. ${ }^{6}$ Additionally, VPS must be immediately removed in an attempt to prevent retrograde spread of the bacteria that may cause ventriculitis. ${ }^{9}$

An unusual complication of VPS-related bowel perforation with extrusion of the shunt through the anus. The case highlights some important considerations of management of this complication. This patient presented with a chronic perforation with no evidence of peritonitis. Our approach for distal catheter removal was to cut the catheter over the chest wall and pull from the distal end protruding from the anus to reduce the risk of spreading bacteria back into the peritoneal cavity. Next, after removing the VPS, intravenous antibiotics were administered and CSF cultures were followed until sterile. During this time, hydrocephalus was managed by the use of an external ventricular drain.

Bowel perforation related to VPS is an infrequent complication that carries a high morbidity and mortality, if not recognised and promptly treated. The mechanism of bowel perforation related to VPS is still unclear.

\section{REFERENCES}

1. Lifshutz $\mathrm{JI}$, Johnson WD. History of hydrocephalus and its treatments. Neurosurg Focus 2001; 11:E1.
2. Vinchon M, Rekate H, Kulkarni AV. Pediatric hydrocephalus outcomes: A review. Fluids Barriers CNS 2012; 9:18.

3. Low SW, Sein L, Yeo TT, Chou N. Migration of the abdominal catheter of a ventriculoperitoneal shunt into the mouth: A rare presentation. Malays J Med Sci 2010; 17:64-7.

4. Sells CJ, Loeser JD. Peritonitis following perforation of the bowel: A rare complication of a ventriculoperitoneal shunt. J Pediatr 1973; 83:823-4.

5. Yousf MM, Jackson NS, Abbas M, Zimmerman RS, Fleischer DE. Bowel perforation complicating ventriculoperitoneal shunt: Case report and review. Gastrointest Endosc 2003; 58:144-8.

6. Esposito C, Porreca A, Gangemi M, Garipoli V, De Pasquale M. The use of laparoscopy in the diagnosis and treatment of abdominal complications of ventriculoperitoneal shunts in children. Pediatr Surg Int 1998; 13:352-4.

7. Ferreira PR, Bizzi JJ, Amantea SL. Protrusion of ventriculoperitoneal shunt catheter through the anal orifie. A rare abdominal complication. J Pediatr Surg 2005; 40:1509-10.

8. Akcora B, Serarslan $Y$, Sangun O. Bowel perforation and transanal protrusion of a ventriculoperitoneal shunt catheter. Pediatr Neurosurg 2006; 42:129-31.

9. Ibrahim AW. E. coli meningitis as an indicator of intestinal perforation by V-P shunt tube. Neurosurg Rev 1998; 21:194-7.

10. Adeloye A. Protrusion of ventriculoperitoneal shunt through the anus: Report of two cases. East Afr Med J 1997; 74:337-9. 